International Journal of Pure and Applied Mathematics

Volume 109 No. 4 2016, 861-868

ISSN: 1311-8080 (printed version); ISSN: 1314-3395 (on-line version)

url: http://www.ijpam.eu

doi: 10.12732 /ijpam.v109i4.9

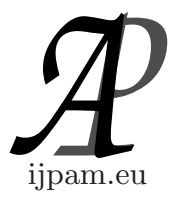

\title{
$J$-CLASS SEMIGROUP OPERATORS
}

\author{
A. Tajmouati ${ }^{1}$, M. El Berrag ${ }^{2}$ \\ ${ }^{1,2}$ Faculty of Sciences \\ Sidi Mohamed Ben Abdellah University \\ Dhar El Mahraz Fez, MOROCCO
}

\begin{abstract}
A $C_{0}$-semigroup $\mathcal{T}=\left(T_{t}\right)_{t \geq 0}$ on an infinite-dimensional separable complex Banach space $X$ is called subspace-hypercyclic for a subspace $M$, if $\operatorname{Or} b(\mathcal{T}, x) \bigcap M$ is dense in $M$ for a vector $x \in M$. In this paper, we localize the notion of M-extended semigroup(resp. M-extended semigroup mixing) limit set of $x$ under $\mathcal{T}$ and We give sufficient conditions of being $M$-hypercyclic for this semigroup. Then by this result, we prove that $\left(T_{t}^{-1}\right)_{t \geq 0}$ is a $M$-hypercyclic. This result is an answer of the question of B. F. Madore and R. A. MartnezAvendano for $C_{0}$-semigroup.
\end{abstract}

AMS Subject Classification: 47C03, 47A10, 47A11

Key Words: $C_{0}$-semigroup, subspace-hypercyclic, subspace-topologically transitive, $J$-class semigroup, $J^{m i x}$-class semigroup

\section{Introduction}

For an infinite-dimensional separable complex Banach space $X, \mathcal{B}(X)$ will denote the algebra of all bounded linear operators on $X$. For $x \in X$, the orbit of $x$ under $\left(T_{n}\right)_{n} \subset \mathcal{B}(X)$ is the set $\operatorname{Orb}\left(T_{n}, x\right)=\left\{T_{n} x: n \in \mathbb{Z}_{+}\right\}$. A sequence $\left(T_{n}\right)_{n}$ of operators is called a hypercyclic, if there is some $x$ whose orbit under $\left(T_{n}\right)_{n}$ is dense in $X$. In such a case, $x$ is called a hypercyclic or universal vector for $\left(T_{n}\right)_{n}$. A sequence $\left(T_{n}\right)_{n}$ of operators is called topologically transitive, if for every nonempty open subsets $U$ and $V$ of $X$, there is some $n \geq 0$ such that

Received: June 12, 2016

Revised: $\quad$ September 3, 2016

Published: $\quad$ October 7, 2016

${ }^{\S}$ Correspondence author (c) 2016 Academic Publications, Ltd. url: www.acadpubl.eu 
$T_{n}(U) \cap V \neq \emptyset$. For some sources on these topics, see [1], [3], [8], [10], [12], [16], [18].

The notions of the limit and extended limit sets are well known in the theory of topological dynamics, see [4]. It is not difficult to show that $T$ is topologically transitive if and only if $J(x)=X$ for every $x \in X$ and that $T$ is topologically mixing if, and only if $J^{\operatorname{mix}}(x)=X$ for every $x \in X$, see [6]. For more information on the $J$-class set, see [2], [5], [6].

The first example of a hypercyclic operator was shown by Birkhoff for the translation operator, Rolewicz constructed the first example of operators on Banach space considering $B$ the backward shift on $l^{p}$, he showed that $\lambda B$ is hypercyclic if, and only if $|\lambda|>1$ (see [3]). Ansari [1] showed that hypercyclic operators exist on infinite-dimensional separable Banach space.

Recall that a one-parameter family $\left(T_{t}\right)_{t \geq 0}$ of operators on $X$ is called a strongly continuous semigroup (or $C_{0}$-semigroup) of operators, if $T_{0}=I, T_{t+s}=$ $T_{t} T_{s}$ for all $t, s \geq 0$ and $\lim _{t \longrightarrow s} T_{t}(x)=T_{s}(x)$ for all $s \geq 0$ and $x \in X$, see [7], [10], [14].

In 2011, B. F. Madore and R. A. Martnez-Avendano in [13] introduced and studied the concept of subspace-hypercyclicity for an operator. An operator $T$ is subspace-hypercyclic or $M$-hypercyclic for a subspace $M$ of $X$, if there exists $x \in X$ such that $\operatorname{Orb}(T, x) \bigcap M$ is dense in $M$. Such a vector $x$ is called a $M$-hypercyclic vector for $T$, they showed that there are operators which are $M$-hypercyclic but not hypercyclic. They introduced analogously the concept of subspace-transitivity. Let $T \in \mathcal{B}(X)$ and $M$ be a closed subspace of $X$, we say that $T$ is $M$-transitive, if for any non-empty open sets $U, V$ in $M$, there exists $n \geq 0$ such that $T^{-n}(U) \bigcap V$ contain a non-empty open subset of $M$. The authors showed that $M$-transitivity implies $M$-hypercyclicity. Note that the converse is not true, this is proven recently by C. M. Le in [11]; for more information see [9], [15]. In 2013 S.Talebi, M.Asadipour localized the notion of subspace-transitivity and gave the answer of the question asked by B.F. Madore and R.A. Martnez-Avendano, see [19].

Recently, in 2015 Abdelaziz Tajmouati, Abdeslam El Bakkali and Ahmed Toukmati introduced and studied the $M$-Hypercyclicity of $C_{0}$-semigroup $\mathcal{T}=$ $\left(T_{t}\right)_{t \geq 0}$ on an infinite-dimensional separable complex Banach space $X$ and gave sufficient conditions of being $M$-hypercyclic for this semigroup. Moreover, some proprieties and analogous results for the notion of $M$-transitive, see [17].

In this paper, we will localize at first the notion of M-extended semigroup(resp. $M$-extended semigroup mixing) limit set of $x$ under $\mathcal{T}$ and We will give sufficient conditions of being $M$-hypercyclic for this semigroup. Then by this result, we will prove that $\left(T_{t}^{-1}\right)_{t \geq 0}$ is a $M$-hypercyclic. This result is an 
answer of the question $(i)$ of B. F. Madore and R. A. Martnez-Avendano for $C_{0}$-semigroup, see $[13]$.

\section{Main Results}

We will assume that the subspace $\mathcal{M} \subset X$ is topologically closed. We start with our main definitions.

Definition 2.1. [17] Let $\mathcal{T}=\left(T_{t}\right)_{t \geq 0}$ be a $C_{0}$-semigroup and $M$ be a nonzero subspace of $X$. We say that $\mathcal{T}$ is $M$-hypercyclic if there exists a vector $x \in X$ such that $\operatorname{Orb}(\mathcal{T}, x) \bigcap M$ is dense in $M$ with $\operatorname{Orb}(\mathcal{T}, x)=\left\{T_{t} x: t \geq 0\right\}$

Definition 2.2. [17] Let $\mathcal{T}=\left(T_{t}\right)_{t \geq 0}$ be a $C_{0}$-semigroup and $M$ be a nonzero subspace of $X$. We call that $\mathcal{T}$ is $M$-transitive if for every tow open, non-empty subsets $U, V$ of $M$ there is $t \geq 0$ such that $T_{t}^{-1}(U) \cap V$ contains a non-empty open set of $M$.

Definition 2.3. Let $\mathcal{T}=\left(T_{t}\right)_{t \geq 0}$ be a $C_{0}$-semigroup and $M$ be a nonzero subspace of $X$. We call that $\mathcal{T}$ is $M$-mixing if for every tow open, non-empty subsets $U, V$ of $M$ there is $t_{0} \geq 0$ such that for all $t \geq t_{0}, T_{t}^{-1}(U) \bigcap V$ contains a non-empty open set of $M$

Theorem 2.1. Let $\mathcal{T}=\left(T_{t}\right)_{t \geq 0}$ be a $C_{0}$-semigroup and $M$ be a nonzero subspace of $X$. Then the following conditions are equivalent

1. $\mathcal{T}$ is $M$-mixing.

2. For every non-empty open $U$ and $V$ of $M$, there is $t_{0} \geq 0$ such that for all $t \geq t_{0}, T_{t}^{-1}(U) \bigcap V$ is a non-empty open of $M$.

3. For every non-empty open $U$ and $V$ of $M$, there is $t_{0} \geq 0$ such that for all $t \geq t_{0}, T_{t}^{-1}(U) \bigcap V$ is non-empty and $T_{t}(M) \subset M$.

Proof. $(2) \Leftrightarrow(1)$ is clair.

$(3) \Rightarrow(2)$. Let $U$ and $V$ be nonempty open subsets of $M$, by (3) there is $t_{0} \geq 0$ such that for all $t \geq t_{0}, T_{t}^{-1}(U) \bigcap V$ is non-empty and $T_{t}(M) \subset M$.

Since $T_{t \mid M}: M \longrightarrow M$ is continuous, then $T_{t}^{-1}(U)$ is open in $M$, therefore $T_{t}^{-1}(U) \cap V$ is nonempty open of $M$.

$(1) \Rightarrow(3)$. Let $U$ and $V$ be tow nonempty open subsets of $M$. By (1) there exists $t_{0} \geq 0$ such that for all $t \geq t_{0}, T_{t}^{-1}(U) \cap V$ contains a nonempty open $W$ of $M$, it follows that $W \subset T_{t}^{-1}(U) \cap V$ and $T_{t}^{-1}(U) \cap V \neq \phi$. 
Next, We prove that $T_{t}(M) \subset M$.

Let $x \in M$, we have $W \subset T_{t}^{-1}(U) \cap V$, this implies that $T_{t}(W) \subset U \subset M$. Let $x_{0} \in W$, since $W$ is open of $M$ then for all $r$ enough small we have $x_{0}+r x \in$ $W$, therefore $T_{t}\left(x_{0}+r x\right)=T_{t} x_{0}+r T_{t} x \in T_{t}(W) \in M$. From $T_{t} x_{0} \in M$ it follows that $T_{t} x \in M$.

We then conclude that $T_{t}(M) \subset M$.

Corollairy 2.1. Let $\mathcal{T}=\left(T_{t}\right)_{t \geq 0}$ be a $C_{0}$-semigroup and $M$ be a nonzero subspace of $X$. If $\mathcal{T}$ is $M$-mixing then $\mathcal{T}$ is $M$-hypercyclic.

Definition 2.4. Let $\mathcal{T}=\left(T_{t}\right)_{t \geq 0}$ be a $C_{0}$-semigroup and $M$ be a nonzero subspace of $X$.

$J\left(T_{t}, M, x\right)=\{y \in X:$ for every relatively open neighborhoods $U, V$ of $x, y$ in $M$ respectively, and there exists $t \geq 0$ such that,$T_{t}(U) \cap V \neq \emptyset$ and $T_{t}(M) \subset$ $M\}, J^{\operatorname{mix}}\left(T_{t}, M, x\right)=\{y \in X$ : for every relatively open neighborhoods $U, V$ of $x, y$ in $M$ respectively, and every $t \geq t_{0}, T_{t}(U) \cap V \neq \emptyset$ and $T_{t}(M) \subset M$ for some $\left.t_{0} \geq 0\right\}$ denote the $M$-extended semigroup(resp $M$-extended semigroup mixing) limit set of $x$ under $\left(T_{t}\right)_{t \geq 0}$.

Theorem 2.2. Let $\mathcal{T}=\left(T_{t}\right)_{t \geq 0}$ be a $C_{0}$-semigroup and $M$ be a nonzero subspace of $X$. Then the following conditions are equivalent

1. $\mathcal{T}$ is $M$-mixing.

2. For every $x \in M, J^{\text {mix }}(\mathcal{T}, M, x)=M$.

Proof. We prove at first that (1) implies (2). Let $x \in U$ and $y \in V$ and $U, V$ be nonempty relatively open subsets of $M$. Then by [3, Theorem 2.1] there is $t_{0} \geq 0$ such that for all $t \geq t_{0}, U \cap T_{t}(V)$ is non-empty and $T_{t}(M) \subset M$. Thus $y \in J^{\operatorname{mix}}(\mathcal{T}, M, x)$, and consequently $J^{\operatorname{mix}}(\mathcal{T}, M, x)=M$.

Now we prove that $(2) \Rightarrow(1)$. Let $U \subset M, V \subset M$, both nonempty and relatively open. We consider $x_{0} \in U, y_{0} \in V$, and since $J^{\operatorname{mix}}\left(\mathcal{T}, M, x_{0}\right)=M$, then there exists $t_{0} \geq 0$ such that for all $t \geq t_{0} T_{t_{0}}^{-1}(V) \cap U \neq \emptyset$ and $T_{t_{0}}(M) \subset M$. Theorem 2.1 implies that $\mathcal{T}$ is $M$-mixing.

Theorem 2.3. Let $\mathcal{T}=\left(T_{t}\right)_{t \geq 0}$ be a $C_{0}$-semigroup and $M$ be a nonzero subspace of $X$. Then the following conditions are equivalent

1. $\mathcal{T}$ is $M$-transitive.

2. For every $x \in M, J(\mathcal{T}, M, x)=M$. 
Proof. We first prove that (1) implies (2). Let $U$ and $V$ be nonempty relatively open subsets of M. Then there exists $t_{0} \geq 0$ such that $T_{t_{0}}^{-1}(U) \cap V$ contains a nonempty open $W$ of $M$, it follows that $W \subset T_{t_{0}}^{-1}(U) \cap V$ and $T_{t_{0}}^{-1}(U) \cap V \neq \phi$.

Next, We prove that $T_{t_{0}}(M) \subset M$.

Let $x \in M$, we have $W \subset T_{t_{0}}^{-1}(U) \cap V$, this implies that $T_{t_{0}}(W) \subset U \subset M$. Let $x_{0} \in W$, since $W$ is open of $M$ then for all $r$ enough small we have $x_{0}+r x \in$ $W$, therefore $T_{t_{0}}\left(x_{0}+r x\right)=T_{t_{0}} x_{0}+r T_{t_{0}} x \in T_{t_{0}}(W) \in M$. From $T_{t_{0}} x_{0} \in M$ it follows that $T_{t_{0}} x \in M$.

We then conclude that $T_{t_{0}}(M) \subset M$. Therefore $J(\mathcal{T}, M, x)=M$, For every $x \in M$.

Now we prove that $(2) \Rightarrow(1)$. Let $U \subset M, V \subset M$, both nonempty and relatively open. We consider $x_{0} \in U, y_{0} \in V$, and since $J\left(\mathcal{T}, M, y_{0}\right)=M$, then there exists $t_{0} \geq 0$ such that $T_{t_{0}}^{-1}(U) \cap V \neq \phi$ and $T_{t_{0}}(M) \subset M$.

Since $T_{t_{0} \mid M}: M \longrightarrow M$ is continuous, then $T_{t_{0}}^{-1}(U)$ is open in $M$, therefore $T_{t_{0}}^{-1}(U) \cap V$ is a relatively open nonempty subset of $M$.

Theorem 2.4. Let $\mathcal{T}=\left(T_{t}\right)_{t \geq 0}$ be a $C_{0}$-semigroup and $M$-mixing. Let $M$ be a nonzero subspace of $X$ and $T_{t}, t \geq 0$ be an invertible operators. Then $\mathcal{T}^{-1}=\left(T_{t}^{-1}\right)_{t \geq 0}$ is M-hypercyclic.

Proof. Let $x, y \in M$ and $U, V$ are relatively open subsets of $M$ such that contain $x, y$ respectively. Then by Theorem 2.2 and the invertibility of $\mathcal{T}=$ $\left(T_{t}\right)_{t \geq 0}$ imply that there exists $t_{0} \geq 0$ such that for all $t \geq t_{0}$,

$$
U \cap T_{t}^{-1}(V) \neq \emptyset \text { and } T_{t}^{-1}(M) \subset M
$$

Hence for every $x \in M, x \in J^{m i x}\left(T_{t}^{-1}, M, y\right)$ and consequently;

$$
\forall y \in M, M=J^{m i x}\left(T_{t}^{-1}, M, y\right)
$$

or equivalently $\mathcal{T}^{-1}$ is M-mixing. by corollary $2.1, \mathcal{T}^{-1}$ is $M$-hypercyclic.

Definition 2.5. Let $\left(T_{n}\right)_{n \geq 0} \subset \mathcal{B}(X)$ be a sequence of operators and $M$ be a nonzero subspace of $X$.

$J\left(T_{n}, M, x\right)=\{y \in X$ : there exist a strictly increasing sequence of positive integers $k_{n}$ and a sequence $x_{n} \subset X$ such that $x_{n} \rightarrow x$ and $T_{k_{n}} x_{n} \rightarrow y$ and for every $\left.n, T_{k_{n}}(M) \subset M\right\}$

$J\left(T_{n}, M, x\right)=\{y \in X$ : for every relatively open neighborhoods $U, V$ of $x, y$ in $M$ respectively, and every positive integer $N \geq 0$, there exists $n>N$ such that , $T_{n}(U) \cap V \neq \emptyset$ and $\left.T_{n}(M) \subset M\right\}$ 
$J^{\text {mix }}\left(T_{n}, M, x\right)=\{y \in X:$ for every relatively open neighborhoods $U, V$ of $x, y$ in $M$ respectively, and every positive integer $n>N, T_{n}(U) \cap V \neq \emptyset$ and $T_{n}(M) \subset M$ for some $\left.N \in \mathbb{N}\right\}$

denote the $M$-extended sequence(resp M-extended sequence mixing) limit set of $x$ under $\left(T_{n}\right)_{n \in \mathbb{N}}$.

Proposition 2.1. An equivalent definition for the sets $J\left(T_{n}, M, x\right)$ is the following:

$J\left(T_{n}, M, x\right)=\{y \in X$ : there exist a strictly increasing sequence of positive integers $k_{n}$ and a sequence $x_{n} \subset X$ such that $x_{n} \rightarrow x$ and $T_{k_{n}} x_{n} \rightarrow y$ and for every $\left.n, T_{k_{n}}(M) \subset M\right\}$

Proof. Let $y \in J\left(T_{n}, M, x\right)$ and consider the open balls $U_{n}=B\left(x, \frac{1}{n}\right) \cap$ $M, V_{n}=B\left(y, \frac{1}{n}\right) \cap M$ centered at $x, y \in X$ and radius $1 / n$ for $n=1,2, \ldots$ and $N=k_{n-1}, k_{0}=1$. Then there exists $k_{n}>N=k_{n-1}$ such that

$$
T_{k_{n}}\left(U_{n}\right) \cap V_{n} \neq \emptyset \text { and } T_{k_{n}}(M) \subset M \text {. }
$$

Hence there exists $x_{n} \in U_{n}$ such that $T_{k_{n}} x_{n} \in V_{n}$ and $T_{k_{n}}(M) \subseteq M$. Therefore $\left(k_{n}\right)$ is an strictly increasing sequence of positive integers and $\left(x_{n}\right)$ is a sequence in $M$ such that $x_{n} \rightarrow x$ and $T_{k_{n}} x_{n} \rightarrow y$ and for every $n, T_{k_{n}}(M) \subset M$. The converse is obvious.

Theorem 2.5. Let $\left(T_{n}\right)_{n \geq 1} \subset \mathcal{B}(X)$ be a sequence of operators and $M$ be a nonzero subspace of $X$. Then the following conditions are equivalent:

1. $\left(T_{n}\right)_{n \geq 1}$ is $M$-transitive( $\operatorname{resp} M$-mixing) .

2. For every $x \in M, J\left(T_{n}, M, x\right)=M\left(\operatorname{resp} J^{\text {mix }}\left(T_{n}, M, x\right)=M\right)$.

Proof. In the proof, we use a proofs method of the theorem 2.3(resp theorem 2.1)

The next example will show that $\left(T_{n}\right)_{n \geq 0} \subset \mathcal{B}(X)$ is not hypercyclic but that $J^{\text {mix }}\left(T_{n}, M, x\right)=M$ for every $x \in M$, i.e $\left(T_{n}\right)_{n \geq 0}$ is $M$-mixing.

Example 1. Let $T=2 B$ such that $B$ is the backward shift on $l^{2}$, i.e. for every $x=\left(x_{1}, x_{2}, x_{3}, \ldots\right) \in l^{2}$;

$$
T^{n}\left(x_{1}, x_{2}, x_{3}, \ldots\right)=2^{n}\left(x_{n+1}, x_{n+2}, \ldots\right), n=1,2, \ldots
$$

It is well known that $T_{n}=T^{n} \oplus I: l^{2} \times l^{2} \rightarrow l^{2} \times l^{2}$ is $M$-hypercyclic where $M=l^{2} \times\{0\}$, but $T_{n}=T^{n} \oplus I$ is not hypercyclic, see ([17], and [13]). Suppose 
that $U \subseteq l^{2}, V \subseteq l^{2}$, both relatively open, and $x=\left(x_{1}, x_{2}, x_{3}, \ldots\right) \in U, y=$ $\left(y_{1}, y_{2}, y_{3}, \ldots\right) \in V$, Then $U \times\{0\}$ and $V \times\{0\}$ are two relatively open sets of $M$. Now for all $n \in \mathbb{N}^{*}$ set;

$$
z_{n}=\left(x_{1}, x_{2}, \ldots x_{n-1}, \frac{y_{1}}{2^{n}}, \frac{y_{2}}{2^{n}}, \ldots, \frac{y_{n-1}}{2^{n}}, \frac{y_{1}}{2^{2 n}}, \frac{y_{2}}{2^{2 n}}, \ldots, \frac{y_{n-1}}{2^{2 n}} \ldots\right),
$$

then $z_{n} \rightarrow x$ and $T^{n} z_{n} \rightarrow y$. Hence there exists a non-negative integer $N$ such that;

$$
\forall n \geq N,\left(T^{n} \oplus I\right)(U \times\{0\}) \cap(V \times\{0\}) \neq \emptyset .
$$

Since $T_{n}(M) \subseteq M$, then by using a similar argument as in Theorem 2.5 we conclude that the operator $\left(T_{n}\right)_{n}=\left(T^{n} \oplus I\right)_{n}$ is $M$-mixing.

The next example will show that $\left(T_{n}\right)_{n \geq 0}$ subspace-hypercyclicity does not imply $J^{\text {mix }}\left(T_{n}, M, x\right)=M$, i.e $\left(T_{n}\right)_{n \geq 0}$ does not imply subspace-mixing with respect to $M$.

Example 2. Let $\lambda \in \mathbb{C}$ be of modulus greater than 1 and let $B$ be the backward shift on $l^{2}$. Let $m$ be a positive integer and $M$ be the subspace of $l^{2}$ consisting of all sequences with zero on the first $m$ entries, i.e.

$$
M=\left\{\left\{a_{n}\right\}_{n=0}^{\infty}: a_{n}=0 \text { for } n \leq m\right\},
$$

then $T_{n}=\lambda^{n} B, n=1,2, \ldots$ is $M$-hypercyclic, see [13]. Now consider

$$
V=\left\{\left\{a_{n}\right\}_{n=0}^{\infty} \in l^{2}: a_{n}=0 \text { for } n \leq m \text { and }\left|a_{n}\right|>0 \text { for } n>m\right\},
$$

so $V$ is relatively open subset of $M$. If $N=m+1$, then for every $n>N, T_{n}(V) \cap$ $M=\emptyset$. Thus for every $x \in M, J^{\operatorname{mix}}\left(T_{n}, M, x\right) \neq M$ and consequently the operator $\left(T_{n}\right)_{n \geq 1}$ is not a $M-$ mixing.

Theorem 2.6. Let $T=\left(T_{n}\right)_{n}$ be a sequence of $\mathcal{B}(X)$ and $M$-mixing. Let $M$ be a nonzero subspace of $X$ and $T_{n}, n=1,2, \ldots$, be an invertible operators. Then $T^{-1}=\left(T_{n}^{-1}\right)_{n}$, is $M$-hypercyclic.

Proof. Similar to theorem 2.4

\section{References}

[1] S.I. Ansari, Existance of hypercyclic operatos on topological vector space, J.F. Anal., 148 (1997), 384-390.

[2] M.R. Azimi, V. Muller, A note on J-sets of linear operators, Revista de la Real Academia de Ciencias Exactass, Fisicas y Naturales, 105, No. 2 (2011), 449-453. 
[3] F. Bayart and E. Matheron, Dynamics of Linear Operators, Cambridge University Press (2009).

[4] N.P. Bhatia and G.P. Szegö, Stability Theory of Dynamical Systems, Die Grundlehren der mathematischen Wissenschaften, Band 161 Springer-Verlag, New York-Berlin (1970).

[5] G. Costakis, A. Manoussos, J-class weighted shifts on the space of bounded sequences of complex numbers, Integral Equations Operator Theory, 62, No. 2 (2008), 149-158.

[6] G. Costakis, A. Manoussos, J-class operators and hypercyclicity, J. Operator Theory, 67, No. 1 (2012), 101-119.

[7] K.J. Engel, R. Nagel, One-Parameter Semigroups for Linear Evolution Equations, Grad. Texts in Math., vol. 194, Springer-Verlag, New York (2000).

[8] K. Goswin and G. Erdmann, Universal families and hypercyclic operators, Bulletin of the American Mathematical Society, 35 (1999), 345-381.

[9] R.R. Jiménez-Munguía, R.A. Martnez-Avendao, A. Peris, Some questions about subspace hypercyclic operators, J. Math. Anal. Appl., 408 (2013), 209-212.

[10] Karl-G. Grosse-Erdmann, Alfred Peris Manguillot, Linear Chaos, pringer-Verlag London Limited (2011).

[11] C.M. Le, On Subspace-hypercyclicity operators, Proc. Amer. Math. Soc., 139, No. 8 (2011), 2847-2852.

[12] F. Leon-Saavedra, V. Muller, Hypercyclic sequences of operators, Studia Math., 175, No. 1 (2006), 1-18.

[13] B.F. Madore, R.A. Martnez-Avendano, Subspace hypercyclicity, J. Math. Anal. Appl., 373 (2011), 502-511.

[14] A. Pazy, Semigroups of Linear Operators and Applications to Partial Differential Equations. Appl. Math. Sci., vol. 44, Springer-Verlag, New York (1992).

[15] H. Rezaei, Notes on subspace-hypercyclic operators, S.J. Math. Anal. Appl., 397 (2013), 428-433.

[16] M.D. Rosa, C. Read, A hypercyclic operator whose direct sum is not hypercyclic, J. Operator Theory, 61 (2009), 369-380.

[17] A. Tajmouati, A. El Bakkali, A. Toukmati, On M-hypercyclic semigroup, Int. J. Math. Anal., 9, No. 9 (2015) 417-428.

[18] A. Tajmouati, M. El berrag, Some results on hypercyclicity of tuple of operators, Italian Journal of Pure and Applied Mathematics, 35 (2015), 487-492.

[19] S. Talebi, M. Asadipour, On subspace-transitive operators, Int. J. of Pure and Appl. Math., 84, No. 5 (2013), 643-649. 